\title{
IMPACTO DA INFORMAÇÃO (CONVENCIONAL E SEM LACTOSE) NA ACEITABILIDADE DE BEBIDAS ACHOCOLATADAS
}

\section{Impact of information (conventional and lactose free) on the acceptance of chocolate beverages}

\author{
Letícia Queli Garcia ${ }^{1 *}$, Mariana Borges de Lima Dutra ${ }^{l}$, Ana Flávia Crispim Mariano ${ }^{l}$, \\ Michely Harumi Miyamoto ${ }^{1}$, Iara Machado Annechini ${ }^{l}$
}

\section{RESUMO}

Através do estudo do comportamento do consumidor em relação a alimentos e bebidas, pode-se encontrar diversos fatores que influenciam diretamente na percepção das características sensoriais de um determinado produto. A expectativa de como um alimento deve ser com relação às informações contidas em seu rótulo é um dos principais fatores que alteram essas percepções. Os alimentos sem lactose foram criados para atenderem uma demanda de consumidores que não podem consumir alimentos com esse tipo de açúcar, mas sem a intenção de modificar seu sabor. A partir deste conceito, o objetivo do trabalho foi verificar se existe diferença significativa entre bebidas achocolatadas, com e sem lactose, com relação aos seus aspectos sensoriais, identificando qual delas se aproxima mais da doçura e sabor de chocolate ideais, a fim de verificar se a informação 'sem lactose' causa mudança de comportamento dos consumidores e como isso interfere na intenção de compra.

Palavras-chave: comportamento; expectativa; rotulagem geral de alimentos; teste sensorial de aceitação.

\begin{abstract}
Through the study of consumer behavior concerning food and beverages, one can find several factors that directly influence the perception of the sensory

1 Instituto Federal do Sul de Minas Gerais, Campus Inconfidentes, Praça Tiradentes, 416, Centro, 37576 000, Inconfidentes, MG, Brasil. E-mail: leticia.queli01@outlook.com

* Autor para correspondência
\end{abstract}

Recebido / Received: 17/07/2020

Aprovado / Approved: 14/10/2020 
characteristics of a given product. The expectation of how a product should be in relation to the information contained on its label is one of the main factors that change these perceptions. Lactose-free foods were created to meet the demand of consumers who cannot consume foods with this type of sugar, but without the intention of changing their taste. Based on this concept, the objective of the work was to verify if there is a significant difference between chocolate beverages, with and without lactose, regarding their sensory aspects, identifying which of them is closer to the ideal sweetness and chocolate flavor, to verify if the 'lactose-free' information causes a change in consumer behavior and how it interferes with purchase intent.

Keywords: behavior; expectancy; general food labeling; sensory acceptance test.

\section{INTRODUÇÃO}

Em 2016, o Brasil foi o $4^{\circ}$ maior produtor mundial de leite. Neste ano foram produzidos 33,62 bilhões de litros de leite, representando aproximadamente $5,1 \%$ da produção mundial. Minas Gerais foi o estado brasileiro de maior produção, representando 26,7\% da produção nacional (MILKPOINT, 2017; FAO, 2018; IPECE, 2018). O setor lácteo é muito importante economicamente, pois gera empregos desde o âmbito rural, de pequenos a grandes produtores, até o industrial onde se desdobra no processamento de diversos produtos.

O leite e os produtos lácteos são fontes de cálcio, proteínas, potássio, fósforo, riboflavina (vitamina B2), magnésio e zinco, sendo muito consumido principalmente pelo seu alto teor de cálcio que é relacionado à prevenção de osteoporose (TÉO, 2002). Apesar do seu alto valor nutricional contribuir para que este mercado se mantenha estável, é, ainda preciso buscar novos consumidores através da mudança ou aprimoramento dos alimentos industrializados, para que além de nutritivos possam atender outras necessidades como possibilitar que grupos com restrições possam consumir um determinado alimento.

A bebida achocolatada é uma bebida láctea e, segundo a Instrução Normativa $n^{\circ}$
16 de 23 de agosto de 2005, bebida láctea é o produto obtido da mistura de leite, soro de leite adicionado ou não de outros produtos ou substâncias alimentícias, onde a base láctea seja no mínimo $51 \%$ do total de ingredientes da bebida (BRASIL, 2005).

Há um aumento do consumo de bebidas à base de soro no Brasil. Por causa de seus aspectos nutricionais, sensoriais e praticidade, esse tipo de bebida é consumida por diversos grupos da população, dentre eles o consumo de bebida láctea achocolatada vem se destacando, principalmente para o público infantil (ZACARCHENCO, 2015).

No entanto, cerca de $50 \%$ da população adulta mundial possui intolerância à lactose. (DELL'OSBELA et al., 2017). A intolerância à lactose é uma reação adversa que envolve a digestão da lactose; pessoas intolerantes a lactose não tem a enzima lactase, responsável pela hidrólise da lactose em glicose e galactose. Sem a quebra desse açúcar, o corpo não consegue absorver a lactose, causando sintomas como a diarreia. Existe também a alergia, que está relacionada ao sistema imunológico do indivíduo, mais associada a proteína do leite (PEREIRA et al., 2012).

Existem outras opções para substituição do leite bovino, como o extrato hidrossolúvel de soja, no entanto não há a mesma quantidade de micronutrientes que o leite, principalmente 
de cálcio (HEANEY et al., 2000), sendo necessário que a indústria de alimentos produza produtos que atinjam as demandas nutricionais de grupos específicos (CASÉ et al., 2005).

O leite e seus derivados sem lactose são mais doces que os produtos tradicionais, isso acontece devido à hidrólise da lactose, fazendo com que se consiga atingir até mesmo os consumidores que não possuam restrição a lactose (PEREIRA et al., 2012). Por menores que sejam as alterações sensoriais de um alimento elas são capazes de causar impacto na aceitação do produto, sendo o estudo do comportamento dos consumidores muito importante para dar embasamento de como o produto irá ser aceito no mercado.

O estudo do comportamento do consumidor em relação aos alimentos e bebidas envolve muitas áreas além da ciência e tecnologia de alimentos, como a psicologia. Vários fatores podem influenciar a percepção das características sensoriais de um produto e, dentre eles, a expectativa é um dos de maior importância (NORONHA et al., 2005).

Para avaliar o impacto que as informações do rótulo causam é preciso fazer a avaliação do produto primeiro sem essas informações. O teste cego é realizado com o produto fora de sua embalagem e codificado com algarismos de três dígitos para que o consumidor não tenha nenhum tipo de informação que possa gerar expectativa sobre aquele produto, pois esta expectativa determina o quanto o consumidor acha que vai gostar ou desgostar do produto somente observando essas informações (NORONHA et al., 2005).

Com isso, o objetivo do trabalho foi avaliar se existem diferenças sensoriais entre bebidas lácteas achocolatadas, com e sem lactose, de mesma marca comercial; determinar qual delas se aproxima mais da doçura e do sabor de chocolate ideais; verificar se são causadas diferenças pela informação 'sem lactose' e quanto isso irá interferir na intenção de compra.

\section{MATERIAL E MÉTODOS}

Foram utilizadas duas amostras de bebida achocolatada, de uma mesma marca, adquiridas no comércio da região Sul de Minas Gerais, sendo elas: convencional e sem lactose. As amostras foram avaliadas por 80 consumidores, com idade média de 22 anos, sendo 15 anos a menor idade e 40 anos a maior, e $53,75 \%$ eram mulheres.

As bebidas foram servidas a $6 \pm 2{ }^{\circ} \mathrm{C}$, em copos plásticos descartáveis codificados com algarismos aleatórios de 3 dígitos, com volume de cerca de $30 \mathrm{~mL}$ cada, em cabines individuais e sob luz branca.

$\mathrm{Na}$ primeira sessão (teste cego), os consumidores avaliaram as amostras de bebida achocolatada (convencional e sem lactose). Neste teste os consumidores não tinham informação sobre qual tipo de bebida achocolatada estão avaliando. Na segunda sessão (teste com informação), com os mesmos consumidores, foi realizada a avaliação das amostras de bebida achocolatada servidas juntamente com a informação na ficharesposta se o produto continha lactose ou não.

O teste de aceitação avaliou os termos aparência, aroma, sabor, textura e impressão global através de escala hedônica não estruturada de nove centímetros ancorada nos extremos por "desgostei muitíssimo" e "gostei muitíssimo” (STONE; SIDEL, 2004).

Para avaliar a doçura ideal do achocolatado, o teste do ideal foi realizado empregando- se escala hedônica estruturada de nove pontos ancorada nos extremos "extremamente menos doce que o ideal" e "extremamente mais doce que o ideal" (MEILGAARD et al., 1999). O mesmo procedimento foi adotado para avaliar o sabor de chocolate ideal.

A intenção de compra foi avaliada 
por escala estruturada de cinco pontos de "certamente não compraria" a "certamente compraria” (MEILGAARD et al., 1999).

Os resultados obtidos foram analisados porANOVA/teste Tukey, a 5\% de probabilidade utilizando-se o programa computacional Sensomaker ${ }^{\circledR}$, desenvolvido por Pinheiro et al. (2013). Para o teste intenção de compra foi elaborado um histograma de frequência, através do software Microsoft Excel ${ }^{\circledR}$ versão 2010 .

\section{RESULTADOS E DISCUSSÃO}

Na Tabela 1 encontram-se os resultados obtidos no teste cego; nenhuma das amostras diferiram entre si para nenhum dos atributos. As respostas tiverem médias entre 7 e 8 , que correspondem a gostei moderadamente e gostei muito.

Segundo a lista de ingredientes presente nos rótulos das bebidas achocolatadas, as únicas diferenças nas formulações desses produtos são a presença da enzima lactase no achocolatado sem lactose e o leite em pó integral reconstituído que aparece apenas na bebida convencional, os demais ingredientes são iguais e aparecem na mesma ordem de colocação, ou seja, em iguais proporções.

Em um trabalho realizado por Rensis; Souza (2008), com iogurte integral e light, também em um teste cego, obtiveram médias entre 6 e 8 para todos os atributos em ambas as amostras com nenhuma diferença significativa, comprovando que o iogurte light se assemelha bastante ao produto integral.

$\mathrm{Na}$ Tabela 2 estão apresentados os resultados obtidos no teste identificado. Apenas na aparência não houve diferença significativa, para os demais atributos a amostra com lactose foi a mais aceita. Apesar da diferença significativa, deve-se ressaltar que em todos os atributos as duas amostras apresentaram notas superiores a 7 (gostei moderadamente).

Como eram os mesmos consumidores nos dois testes pode-se observar uma expectativa negativa a informação "Sem Lactose" na bebida achocolatada. A mesma expectativa foi encontrada em um estudo realizado

Tabela 1 - Resultados do teste cego para bebidas lácteas achocolatadas*

\begin{tabular}{cccccc}
\hline Amostras & Aparência & Aroma & Sabor & Textura & Impressão Global \\
\hline Com Lactose & $7,85 \pm 1,23^{\mathrm{a}}$ & $7,54 \pm 1,33^{\mathrm{a}}$ & $7,73 \pm 1,29^{\mathrm{a}}$ & $7,54 \pm 1,37^{\mathrm{a}}$ & $7,46 \pm 1,17^{\mathrm{a}}$ \\
Sem Lactose & $7,70 \pm 1,27^{\mathrm{a}}$ & $7,20 \pm 1,57^{\mathrm{a}}$ & $7,28 \pm 1,67^{\mathrm{a}}$ & $7,39 \pm 1,50^{\mathrm{a}}$ & $7,76 \pm 1,34^{\mathrm{a}}$ \\
\hline
\end{tabular}

* Médias \pm desvio padrão com letras iguais em cada coluna indicam que não há diferença significativa ( $>0,05)$.

Tabela 2 - Resultados do teste identificado para bebidas lácteas achocolatadas*

\begin{tabular}{cccccc}
\hline Amostras & Aparência & Aroma & Sabor & Textura & Impressão Global \\
\hline Com Lactose & $8,03 \pm 1,04^{\mathrm{a}}$ & $7,68 \pm 1,19^{\mathrm{a}}$ & $7,96 \pm 1,06^{\mathrm{a}}$ & $7,86 \pm 1,05^{\mathrm{a}}$ & $7,95 \pm 0,91^{\mathrm{a}}$ \\
Sem Lactose & $7,73 \pm 1,17^{\mathrm{a}}$ & $7,21 \pm 1,55^{\mathrm{b}}$ & $7,18 \pm 1,57^{\mathrm{b}}$ & $7,24 \pm 1,49^{\mathrm{b}}$ & $7,34 \pm 1,65^{\mathrm{b}}$ \\
\hline
\end{tabular}

* Médias \pm desvio padrão com letras iguais em cada coluna indicam que não há diferença significativa $(\mathrm{p}>0,05)$ 
por Dutra et al. (2015), onde avaliaram gelatinas de framboesa, convencional, diet e light, em três sessões com os mesmos consumidores. Na primeira sessão foi feito um teste cego, na segunda, teste somente com a embalagem e na terceira sessão, teste com informação, onde a amostra foi servida com sua respectiva embalagem. Como resultado, a expressão "Light" influenciou negativamente a impressão global desse produto.

Toda expectativa prévia de um produto gerada pelo consumidor, seja ela tanto para melhor como para pior, causará uma negatividade generalizada levando a menor aceitação do produto (NORONHA et al., 2005).

Na Tabela 3 estão apresentados os valores obtidos no teste do ideal para doçura e sabor de chocolate quando codificados. As amostras não se diferiram nem para o quesito doçura nem para o sabor de chocolate, embora a amostra com lactose tenha se aproximado mais do ideal em ambos os aspectos.

Para ambas as amostras pode-se observar o mesmo comportamento. Para doçura, seus valores foram superiores a zero, o que significa que as duas estão mais doces que o ideal e, também, as duas bebidas tem menos sabor de chocolate que o ideal.

Segundo Ramalho; Ganeco (2016), quando se hidrolisa a lactose em glicose e galactose a solubilidade e a doçura do produto aumentam, entretanto, a viscosidade do soro e permeado diminuem. A doçura da lactose hidrolisada é de aproximadamente $70 \%$ da doçura da sacarose, fato este que pode explicar o valor da doçura da amostra sem lactose ser superior ao da amostra com lactose e o contrário para o sabor de chocolate também.

$\mathrm{Na}$ Tabela 4 estão apresentados os valores obtidos no teste do ideal para doçura e sabor de chocolate quando identificados. As amostras não se diferiram com relação ao sabor de chocolate, no entanto houve diferença significativa quanta a doçura, onde a amostra sem lactose foi mais próxima do ideal.

A doçura das amostras também apresentou resultado diferente no teste identificado, sendo a bebida sem lactose a mais próxima do ideal e desta vez menos doce que o ideal, por apresentar um valor negativo. Assim

Tabela 3 - Doçura e sabor de chocolate pelo teste do ideal cego para bebidas lácteas achocolatadas*

\begin{tabular}{lll}
\hline Amostras & Doçura & Sabor de Chocolate \\
\hline Com Lactose & $0,15 \pm 1,07^{\mathrm{a}}$ & $-0,14 \pm 1,23^{\mathrm{a}}$ \\
Sem Lactose & $0,31 \pm 1,23^{\mathrm{a}}$ & $-0,16 \pm 1,34^{\mathrm{a}}$ \\
\hline
\end{tabular}

* Médias \pm desvio padrão com letras iguais em cada coluna indicam que não há diferença significativa ( $>0,05)$.

Tabela 4 - Doçura e sabor de chocolate pelo teste do ideal identificado para bebidas lácteas achocolatadas* $^{*}$

\begin{tabular}{cll}
\hline Amostras & Doçura & Sabor de Chocolate \\
\hline Com Lactose & $0,28 \pm 0,98^{\mathrm{a}}$ & $-0,13 \pm 0,95^{\mathrm{a}}$ \\
Sem Lactose & $-0,11 \pm 1,13^{\mathrm{b}}$ & $-0,43 \pm 1,06^{\mathrm{a}}$ \\
\hline * Médias \pm desvio padrão com letras iguais em cada coluna indicam que não há diferença significativa (p $>0,05)$.
\end{tabular}


como no teste cego, no teste com informação a amostra com lactose foi mais próxima do ideal quanto ao sabor de chocolate, entretanto a bebida sem lactose se distanciou mais do ideal neste quesito quando identificada, resultado este que se assemelha a mudança de comportamento na avaliação sensorial.

No histograma de intenção de compra (Figura 1) pode-se observar que houve uma mudança na decisão dos consumidores. No teste cego a amostra com lactose teve $78,75 \%$ de frequência na intenção de compra positiva e $2,5 \%$ de intenção de compra negativa, enquanto no teste com informação houve um aumento na frequência de intenção de compra positiva, $86,25 \%$ e, uma queda na intenção de compra negativa para $1,25 \%$. Para a amostra sem lactose aconteceu o contrário, houve uma queda na frequência de compra positiva, de $58,75 \%$ para $57,5 \%$, e um aumento frequência negativa quando identificadas de $10 \%$ para $15 \%$.

Uma pesquisa realizada por Della Lucia et al. (2007), em que foi feita a avaliação da aceitação sensorial de uma embalagem de café contendo apenas a informação que o produto era orgânico e nada mais, constatou que essa informação (orgânico) influenciou na intenção de compra de quase $80 \%$ dos consumidores.

Almeida et al. (2016) realizaram um estudo com sorvete de mangaba sem lactose e diet e verificaram que $77 \%$ dos provadores detectaram diferenças nas formulações, mas ainda assim $43 \%$ deles preferem a formulação sem lactose e diet, assim, concluíram que os produtos sem lactose e diet são formulados de maneira a se aproximarem muito dos produtos tradicionais, o que facilita a aceitação dos consumidores.

Apesar das mudanças na intenção de compra, pode-se dizer que o comportamento dos consumidores com relação as amostras foram muito similares em ambos os testes. Deve-se destacar que somente a bebida sem lactose teve respostas 'certamente não compraria' e esse número não se alterou de um teste para outro, representando $1,25 \%$, o que sugere que esses consumidores não foram influenciados pela informação da amostra e sim por seus fatores intrínsecos.

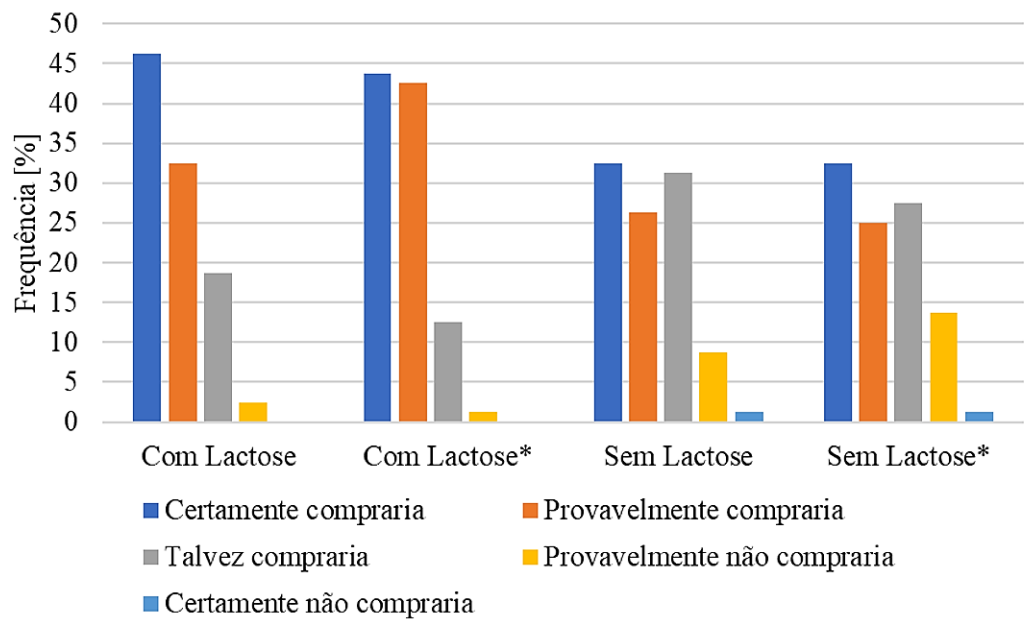

* análise identificada

Figura 1 - Gráfico da intenção de compra das amostras de achocolatado com e sem lactose na análise sensorial codificada e identificada 


\section{CONCLUSÃO}

As bebidas achocolatadas não apresentaram diferenças sensoriais significativas, sendo consideradas, também, muito próximas em níveis de doçura e sabor de chocolate. A informação 'sem lactose' causa uma expectativa negativa na aceitação do produto que leva a mudanças na avaliação dos aspectos intrínsecos da bebida ocasionando um aumento considerável na intenção de compra negativa.

\section{AGRADECIMENTOS}

Agradecemos a Fundação de Amparo à Pesquisa do Estado de Minas Gerais pelo financiamento do projeto e ao Instituto Federal de Educação, Ciência e Tecnologia do Sul de Minas Gerais - Campus Inconfidentes pela infraestrutura cedida para realização do mesmo.

\section{REFERÊNCIAS}

ALMEIDA, A. B. S. et al. Elaboração e avaliação sensorial de sorvete diet e sem lactose de mangaba endêmica do Cerrado. Revista de Agricultura Neotropical, v. 3, n. 3, p. 38-41, 2016. DOI: 10324/rean.v3i3.1206

BRASIL. Ministério da Agricultura, Pecuária e Abastecimento. Instrução Normativa $\mathrm{n}^{\mathrm{o}} 16$, de 23 de agosto de 2005. Aprova o Regulamento Técnico de Identidade e Qualidade de Bebida Láctea. Diário Oficial da União: seção 1, Brasília, DF, n.163, p. 7, 24 ago. 2005.

CASÉ, F. et al. Produção de 'leite' de soja enriquecido com cálcio. Ciência e Tecnologia de Alimentos, v. 1, n. 25, p. 86-91, 2005.

DELL'OSBELA, R. S. et al. Desenvolvimento de produto para intolerantes à lactose: Produção de sorvete de goiaba. In: CONGRESSO DE PESQUISA E EXTENSÃO DA FSG, 5.; SALÃO DE EXTENSÃO, 3., 2017, Caxias do Sul. Anais[...]. Caxias do Sul: Faculdade da Serra Gaúcha, 2017. p. 243-245.

DELLA LUCIA, S. M. et al. Fatores da embalagem de café orgânico torrado e moído na intenção de compra do consumidor. Ciência e Tecnologia de Alimentos, v. 27, n. 3, p. 485-491, 2007.

DUTRA, M. B. L. et al. Influência da informação (convencional, diet e light) na aceitação sensorial de gelatina sabor framboesa. Brazilian Journal of Food Research, v. 6, n. 2, p.10-14, 2015. DOI: 10.14685/rebrapa.v6i2.147

FAO - FOOD AND AGRICULTURE ORGANiZATION. Livestock Primary. 2018. Disponível em: http://www.fao.org/ faostat/en/\#data/QL. Acesso em 15/09/2018.

HEANEY, R. P. et al. Bioavailability of the calcium in fortified soy imitation milk, with some observations on method. The American Journal of Clinical Nutrition, v. 71, n. 5, p. 1166-1169, 2000. DOI: 10.1093/ ajen/71.5.1166

IPECE - INSTITUTO DE PESQUISA E ESTRATÉGIA ECONÔMICA DO CEARÁ. Análise da cadeia produtiva do leite e seus derivados no Ceará. IPECE Informe, n. 128, p. 1-27, 2018. Disponível em: https:// www.ipece.ce.gov.br/wp-content/uploads/ sites/45/2018/09/ipece_informe_128_30 Maio_2018.pdf Acesso em: 15 set. 2018.

MEILGAARD, M.; CIVILLE, G. V.; CARR, B. T. Sensory Evaluation Techniques. 3. ed. Boca Raton: CRC Press, 1999. 354 p.

MICROSOFT OFFICE ${ }^{\circledR}$ Excel 2010. 
Disponível em: http://www.office.microsoft. com/pt-br/store Acesso em: 18 de março de 2018 .

MILK POINT. IBGE: Produção de leite cai 2,9\% em 2016; pesquisa aponta aumento dos rebanhos bovinos. 2017. Disponível em: https://www.milkpoint.com.br/noticiase-mercado/giro-noticias/ibge-producaode-leite-cai-29-em-2016-pesquisa-apontaaumento-dos-rebanhos-bovinos-107461n. aspx?r=882017502\#. Acesso em: 03 jul. 2018.

NORONHA, R. L. F.; DELIZA, R.; SILVA. M. A. A. P. A expectativa do consumidor e seus efeitos na avaliação sensorial e aceitação de produtos alimentícios. Alimentos e Nutrição, v. 16, n. 3, p. 299-308, 2005.

PEREIRA, M. C. S. et al. Os lácteos com baixo teor de lactose: Uma necessidade para portadores de má digestão da lactose e um nicho de mercado. Revista Indústria Laticínios, v. 67, n. 389, p. 57-65, 2012.

PINHEIRO, A. C. M.; NUNES, C. A.; VIETORIS, V. Sensomaker: A tool for sensorial characterization. Ciência e Agrotecnologia, v. 37, n. 3, p. 199-201, 2013. DOI: $10.1590 / \mathrm{S} 1413-70542013000300001$

RAMALHO, M. E. O.; GANECO, A. G. Intolerância a lactose e o processamento dos produtos zero lactose. Revista Interface Tecnológica, v. 13, n. 1, p. 119-133, 2016.

RENSIS, C. M. B. V.; SOUZA, P. F. F. Análise sensorial de iogurtes light elaborados com adição de fibras de inulina e oligofrutose. Fazu, v. 1, n. 5, p. 68-72, 2008.

STONE, H.; SIDEL, J. Sensory Evaluation Practices. 3. ed. New York: Academic Press, 408 p. 2004.

TÉO, C. R. P. A. Intolerância à lactose: Uma breve revisão para o cuidado nutricional. Arquivos de Ciência da Saúde da UNIPAR, v. 6, n. 3, p. 135-140, 2002.

ZACARCHENCO, P. B. Bebidas lácteas: Alternativa para nutrição e conveniência. Indústria de Laticínios, n. 112, p. 22-23, 2015. 